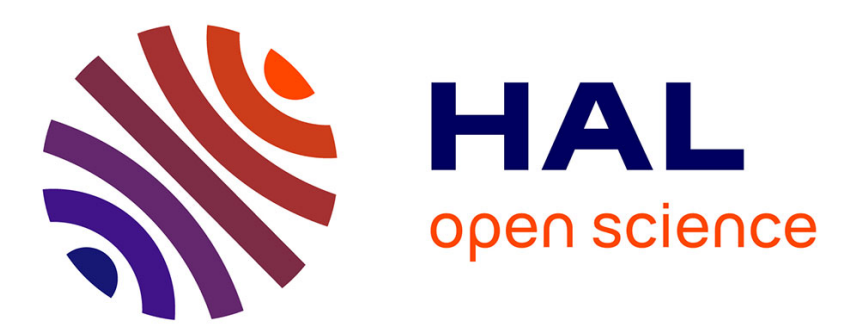

\title{
Soigner sans compter. Les agents de l'hôpital public face à l'épidémie de Covid-19
}

Maud Gelly, Alexis Spire

\section{To cite this version:}

Maud Gelly, Alexis Spire. Soigner sans compter. Les agents de l'hôpital public face à l'épidémie de Covid-19. Revue française des affaires sociales, 2021. halshs-03500079

\section{HAL Id: halshs-03500079 \\ https://shs.hal.science/halshs-03500079}

Submitted on 21 Dec 2021

HAL is a multi-disciplinary open access archive for the deposit and dissemination of scientific research documents, whether they are published or not. The documents may come from teaching and research institutions in France or abroad, or from public or private research centers.
L'archive ouverte pluridisciplinaire HAL, est destinée au dépôt et à la diffusion de documents scientifiques de niveau recherche, publiés ou non, émanant des établissements d'enseignement et de recherche français ou étrangers, des laboratoires publics ou privés. 


\section{Soigner sans compter. Les agents de l'hôpital public face à l'épidémie de Covid-19}

Maud Gelly et Alexis Spire

\section{Résumé}

L'épidémie de Covid-19 survenue en mars 2020 a mobilisé tous les personnels des hôpitaux les plus touchés et a contraint leur administration à suspendre les restrictions budgétaires pour accueillir l'afflux de malades. À partir d'une enquête qualitative menée dans deux hôpitaux d'Îlede-France et du Grand-Est, cette contribution montre ce qu'a représenté ce moment critique pour les personnels de ces institutions. Les auteurs mettent en évidence les ressorts d'un service public qui est confronté à des besoins débordant ses capacités. Dans ce contexte, les personnels ont consenti à fournir un surtravail et à s'investir dans leur mission sans compter, bien au-delà de ce que prévoient les règlements. Cet engagement trouve sa source dans l'intériorisation de la norme du travail bien fait et dans l'attachement à des collectifs de travail qui ont porté les agents durant cette période. Les rapports de domination entre catégories de personnels et entre services hospitaliers n'ont pas pour autant disparu et ont resurgi avec d'autant plus d'acuité une fois la crise passée.

\section{Healing without cost counting. Public hospital staff face the COVID-19 pandemic Abstract}

The COVID-19 pandemic which first reared its head in March 2020 rallied all the staff in the most affected hospitals and forced their administrations to halt budgetary restrictions in favour of admitting the influx of sick people. According to a qualitative study carried out in two Ile-de-France and Grand-Est hospitals, this article shows what this critical moment represented for the staff in these institutions. The authors highlight the resilience of a public service that is confronted with needs that go beyond its capabilities. In this regard, the staff members agreed to take on an overwork and go above and beyond in their mission, past what the regulations usually allow. This commitment is due to the internalisation of the high standard of work performance and the attachment to work collectives that supported the staff members through this period. The relations of domination between categories of staff and between hospital services did not altogether disappear, they resurfaced more sharply once the crisis had passed. 


\section{Introduction}

Depuis plusieurs années, l'hôpital public n'a plus les moyens d'accomplir les missions qui lui sont assignées ${ }^{1}$. Les services des urgences sont devenus le symptôme et l'illustration de cette crise (Belorgey, 2010 ; Morel, 2019). Au lieu de chercher à répondre à l'augmentation des besoins, plusieurs réformes récentes ont été mises en place pour contenir les dépenses. La tarification à l'activité a eu pour conséquence un étranglement budgétaire de l'hôpital public (Juven et Lemoine, 2018), auquel se sont ajoutées des fusions hospitalières (Juven et al., 2019) et des fermetures de lits toujours plus nombreuses (Pierru, 2020).

Ces réformes successives ont fait naître un décalage structurel entre, d'une part, la façon dont les soignants se représentent leur mission et investissent leur métier et, d'autre part, les contraintes que les normes comptables et bureaucratiques de l'institution hospitalière font peser sur le quotidien de leur activité professionnelle. C'est dans ce contexte qu'a déferlé la vague épidémique de Covid-19 au printemps 2020. Dans les hôpitaux les plus touchés, les personnels ont pu se consacrer pleinement aux missions de soins, alors que les directions administratives étaient contraintes de bouleverser de fond en comble toute une série de règles de gestion devenues intangibles.

Pour en prendre la mesure, il faut rompre avec les représentations médiatiques centrées sur les seuls acteurs dominants de l'institution, à savoir les réformateurs et les médecins, et prendre en considération la totalité des personnels soignants (agents de service hospitalier, aides-soignantes, brancardiers $^{2}$, infirmières et médecins) ainsi que les personnels administratifs et techniques. L'enjeu est de montrer que l'épidémie de Covid-19, qui a brutalement replacé les enjeux de santé au cœur du débat public, a aussi constitué un moment fort de socialisation commune pour tous les agents hospitaliers, remettant en question tout un ensemble de logiques bureaucratiques et.

On s'attachera, dans un premier temps, à montrer que l'épidémie de Covid-19, parce qu'elle intervient dans des hôpitaux fonctionnant à flux tendu ${ }^{3}$, c'est-à-dire sans lit vide et en sous-effectif chronique, a contraint les directions hospitalières à rompre avec la logique de rationnement des soins et à s'adapter à l'afflux de malades, en augmentant le travail des personnels et en reportant la majeure partie des interventions et des hospitalisations considérées comme «non urgentes ». Dans un deuxième temps, nous montrerons dans quelles conditions les agents hospitaliers ont consenti à ce surcroît de travail, en se recentrant sur leur mission de soigner et en s'appuyant sur des collectifs de travail renforcés par la crise. Les agents ont pu ainsi soigner sans compter, au double sens du terme : sans compter leur temps, leur énergie et leurs forces pour lutter contre l'épidémie ; sans compter le matériel et les équipements que les directions hospitalières ont mis à leur disposition. Cependant, au-delà du caractère exceptionnel des moyens mis en œuvre, la crise épidémique n'a effacé ni les divisions internes, ni les rapports de domination entre groupes professionnels ; nous montrerons, dans un troisième temps, comment elle a plutôt reformulé les hiérarchies entre services hospitaliers. Loin de se réduire à une parenthèse enchantée, ce moment critique a plutôt fait percevoir aux agents la possibilité de s'émanciper des logiques bureaucratiques et comptables, tout en révélant l'hystérésis des rapports de domination entre catégories de personnels et entre services.

${ }^{1}$ Cet article s'inscrit dans le programme de recherche "Confiance dans les institutions étatiques et scientifiques (CIESCO)», financé dans le cadre de l'appel Flash Covid-19 (Edition 2020) de l'Agence Nationale de la Recherche (ANR).

${ }^{2}$ Les noms des professions majoritairement féminines sur notre terrain sont écrits au féminin et les professions majoritairement masculines au masculin.

${ }^{3}$ Durant la première vague épidémique, ce sont les hôpitaux des régions Île-de-France, Hauts-de-France et Grand-Est qui ont été les plus touchés, tandis que dans ceux des autres régions, comme en Nouvelle-Aquitaine, beaucoup de soignants ont dû attendre des malades qui n'arrivaient pas. 


\section{Encadré 1 Une ethnographie comparée de deux hôpitaux}

Nous avons choisi d'enquêter dans deux groupes hospitaliers qui ont été fortement touchés par l'épidémie de Covid.

L’hôpital A, situé dans le Grand-Est, est le produit de la fusion de plusieurs établissements hospitaliers. Il comprend un hôpital central de court séjour et des hôpitaux de proximité. Ce n'est pas un centre hospitalier universitaire (CHU) mais, avec 6400 agents qui y travaillent, c'est le premier employeur de la région. Au plus fort de la première vague épidémique qui s'est étalée du $1^{\mathrm{er}}$ mars à la fin du mois de mai, près de 500 lits ont été occupés par des patients atteints du Covid.

L’hôpital B, situé en région parisienne, regroupe également plusieurs établissements ; les 4600 agents de ce groupe hospitalier sont répartis sur trois sites et dans 54 services. Il s'agit d'un centre hospitalier universitaire qui affiche une triple mission de soin, d'enseignement et de recherche.

Au total, 93 entretiens ont été réalisés avec des personnels de directions, des chefs de pôle, des chefs de service, des médecins, des infirmières, des aides-soignantes, des cadres de santé, des cadres de pôle, des agents de service hospitalier, des secrétaires, des assistantes sociales et des ergothérapeutes. Nous leur avons présenté l'enquête comme une étude portant sur la façon dont les agents hospitaliers ont été confrontés à l'épidémie. Nous avons essuyé deux refus et treize agents médecins pour la majorité d'entre eux - n'ont pas répondu à nos sollicitations. Parmi les entretiens réalisés, nous avons sélectionné les extraits les plus significatifs de façon à rendre compte des régularités dans les propos recueillis tout au long de l'enquête. Pour situer ces agents au sein de l'espace social, nous avons également pris soin de recueillir et de restituer des éléments de leur trajectoire sociale et professionnelle. Leurs prénoms ont été modifiés, ainsi que les éléments biographiques qui permettaient de les identifier.

Dans chacun de ces entretiens, nous avons recueilli des récits sur les expériences vécues durant la crise épidémique et nous avons posé plusieurs questions sur le détail des activités de travail exercées avant la crise afin de saisir les changements et les continuités des situations de travail au sein de l'institution hospitalière.

\section{L'hôpital public face à un « moment critique »}

La première vague de l'épidémie, en mars 2020, survient alors que l'hôpital public ${ }^{4}$ est soumis, d'un part, aux transformations induites par le virage ambulatoire et, d'autre part, aux tensions dont témoigne une année de mobilisation des infirmières et des aides-soignantes de plusieurs services d'urgence pour dénoncer le déficit de personnels et de lits d'hospitalisation (Collectif Inter-Urgences, 2021). L'afflux massif de malades touchés par une épidémie nouvelle, contagieuse et mortelle se présente comme un «moment critique », c'est-à-dire un moment où, « en rupture avec l'expérience ordinaire du temps comme simple reconduction du passé ou d'un avenir inscrit dans le passé, tout devient possible (au moins en apparence) » (Bourdieu, 1984, p. 236-237). C'est l'affaiblissement provisoire de la logique de rationnement des soins autour de laquelle l'institution hospitalière s'est réorganisée qui conduit les agents à se mobiliser sans compter, dans la perspective d'une issue où tout pourrait changer.

\section{La mobilisation de la force de travail}

\footnotetext{
${ }^{4}$ Dans cet article, on utilise le terme « hôpital public » au singulier pour désigner l'institution au sens générique.
} 
Dans les deux hôpitaux enquêtés, les premières mesures prises par l'administration ont d'abord consisté à augmenter les moyens humains disponibles : outre l'appel médiatisé à la réserve sanitaire, à des étudiants, à des retraités et à des renforts en personnels venus de régions moins touchées par l'épidémie, la principale mesure, beaucoup plus discrète, a été d'augmenter rapidement le temps de travail hebdomadaire de chaque agent.

Le «plan blanc ${ }^{5}$ a d'abord permis d'annuler des interventions non urgentes et de réaffecter les personnels ainsi libérés dans d'autres services. De nombreux personnels soignants ont renoncé à leurs congés ou à leurs jours de repos pour se joindre à l'effort général ; parmi les autres agents, ce retour au travail a aussi pu s'effectuer par la contrainte :

«C'était surtout pour avoir les agents à disposition avec les vagues de congés. Ça leur permet de nous rappeler. [...] Quand tout a commencé, le vendredi 13 mars, je devais être en congés le lendemain. Et à la deuxième vague, pareil, je devais prendre une semaine de congés et je n'ai pu prendre que deux jours. »

Linda, secrétaire dans un service de consultation, hôpital A, 14 janvier 2021.

Le bouleversement des emplois du temps et des congés dépasse largement la sphère professionnelle et touche la plupart des agents dans l'organisation de leur vie privée et familiale qui, le temps de la crise, se trouve (comme dans le cas ci-dessus) arraisonnée à la lutte contre l'épidémie.

La deuxième mesure a été d'imposer le passage à la journée «en 12 heures » à des services qui n'y étaient pas encore astreints ${ }^{6}$. Ce dispositif dérogatoire déjà introduit avant la crise du Covid permet de diminuer le «temps de transmissions », c'est-à-dire le moment d'échange d'informations sur l'état de santé des personnes hospitalisées, considéré par les directions hospitalières comme non productif (Vincent, 2017).

Le troisième levier a consisté à augmenter les heures supplémentaires et le nombre de gardes. L'autre mesure, spécifique à la crise épidémique, a été de maintenir au travail des agents malades du Covid, pour peu que leurs symptômes ne soient pas trop marqués. La direction et les médecins du travail ont justifié cette décision par la nécessité de ne pas désarmer les services par des arrêts de travail trop nombreux :

«Les gens, quand ils étaient vraiment malades, étaient en arrêt de travail. Mais les peu symptomatiques travaillaient. [...] C'est ce qu'on leur disait et heureusement qu'on leur disait ça, sinon la moitié de l'hôpital aurait été absente et je ne sais pas qui aurait soigné les patients Covid. Il faut quand même être réaliste. [...] Il y a eu quelques réunions avec la direction et les médecins internistes qui disaient que si les gens n'étaient pas symptomatiques, même s'ils avaient le PCR positif, ils pouvaient venir travailler à condition de respecter les gestes barrières, de bien mettre leur masque, de ne pas prendre leur pause avec leurs collègues. On n'aurait pas pu fonctionner autrement. »

Médecin du travail, hôpital A, 18 septembre 2020.

Il y avait donc un consensus entre la direction administrative, les médecins du travail et les cadres et chefs de service pour maintenir au travail des agents ayant peu de symptômes, pour que

\footnotetext{
${ }^{5}$ Le «plan blanc» permet à chaque établissement de santé de se doter d'un dispositif de crise, qui lui permet de mobiliser immédiatement les moyens de toute nature pour faire face à une situation sanitaire exceptionnelle.

${ }^{6}$ Dans les services dits «de soins » ou «d'hospitalisation complète », la journée de 24 heures est habituellement découpée en trois tranches, entre lesquelles les agents tournent sept jours sur sept.
} 
les services puissent fonctionner. Ainsi, une fièvre modérée ou une toux pouvaient être considérées comme compatibles avec le travail :

«J'allais travailler avec une toux immense, j'ai fait le test. Ils n'ont pas voulu me le faire à l'hôpital. [...] J'ai dû le faire dans un laboratoire externe, chez moi, et j'ai été détectée positive. [...] Et après, mon chef m'a dit: “Tu mets le masque et tu viens travailler." Parce que, physiquement, j'étais bien, j'avais cette toux qui me gênait un peu. »

Latifa, aide-soignante aux urgences, hôpital A, 15 octobre 2020.

Pour certaines aides-soignantes comme Latifa, cette injonction à travailler, même malades, n'a pas été facile à assumer car elle pouvait induire une mise en danger des patients dont elle s'occupait. D'une certaine manière, le poids de l'autorité hiérarchique a neutralisé ce qui aurait pu constituer un dilemme moral entre la nécessite de contribuer à l'effort collectif et le risque de contaminer les patients.

Face à l'urgence de la crise épidémique, les directions hospitalières sont parvenues à accroître les moyens humains grâce aux renforts en dérogeant aux normes d'éviction du personnel malade et contagieux. Cette solution de court terme a permis de parer au plus pressé et de faire face à l'afflux de malades, au prix d'une intensification du travail à rémunération constante, que l'on peut alors qualifier de surtravail alors même qu'il ne dégage pas de plus-value financière.

\section{L'administration au service de la logique médicale?}

Les mesures prises par l'administration hospitalière pour intensifier le travail montrent que cette dernière ne s'est pas effacée durant la vague épidémique. En revanche, elle a suspendu provisoirement les contraintes budgétaires en acceptant toutes les demandes de matériels, de personnels et de travaux formulées pour lutter contre l'épidémie. Ainsi, le «moment critique » de la crise épidémique a été caractérisé par une plus grande autonomie laissée aux cadres et aux chefs de service :

«Dans les réunions de crise, il y avait dix médecins pour deux administratifs, et ça marchait beaucoup mieux [rires]. C'est-à-dire que toutes les superstructures qu'on avait inventées avant la crise se sont dissoutes. Elles n'avaient aucune utilité dans la gestion de la crise et on est revenu à la seule unité qui avait un sens en termes d'organisation et de décision, à savoir le service hospitalier... Je n'ai jamais eu de freins de la direction en ce qui concerne mes demandes, ce qui était assez extraordinaire parce que, habituellement, pour avoir une infirmière, il faut que j'attende deux ans. Et là, j'ai demandé des heures supplémentaires, des gardes supplémentaires, des moyens supplémentaires, j'ai tout eu tout de suite. C'est une situation rêvée pour un chef de service. »

Fabrice, chef de service des urgences ${ }^{7}, 30$ juin 2020.

Ce que ce praticien hospitalier a particulièrement apprécié dans la gestion de la crise épidémique, c'est que son service des urgences ait pu fonctionner sans avoir à chercher des lits disponibles pour hospitaliser les malades. Ce desserrement des contraintes administratives a d'ailleurs été plus largement ressenti par l'ensemble des médecins et n'a pas manqué de susciter une forme de nostalgie une fois la crise passée :

\footnotetext{
${ }^{7}$ Lorsque les fonctions permettent d'identifier les personnes parce qu'elles sont seules à occuper le poste désigné dans un hôpital donné, nous ne précisons pas de quel hôpital il s'agit.
} 
«Le problème des lits est toujours là et c'est le plus pénible parce que vous passez du temps dessus alors que vous devriez passer du temps auprès du malade. On est beaucoup plus sur l'ordinateur et au téléphone, notre outil premier c'est le téléphone, ce n'est pas le stéthoscope. On passe beaucoup plus de temps à faire de l'administratif, de l'organisationnel, de la logistique que de la médecine en elle-même. »

Hélène, médecin aux urgences, hôpital A, 12 novembre 2020.

Dans les hôpitaux étudiés, la crise épidémique n'a donc pas modifié l'équilibre des pouvoirs entre les autorités administrative et médicale. Tout juste a-t-elle rendu possible une suspension très temporaire de la contrainte budgétaire, en permettant ainsi de soigner sans compter.

\section{La réorganisation des services}

En l'espace de quelques jours, beaucoup de services ont été bouleversés, de façon à libérer des lits pour les malades du Covid et à permettre leur prise en charge sans risquer de contaminer les autres patients. Dans ce contexte, les cadres de santé (sous la responsabilité desquelles sont placées les infirmières et les aides-soignantes) ont joué un rôle central et l'administration a rendu possibles, d'un jour sur l'autre, des réaménagements qui habituellement prennent plusieurs mois.

L'annulation ou le report de toutes les consultations et hospitalisations ne relevant ni des cancers, ni de l'obstétrique, ni des urgences a permis de libérer des lits et du personnel pour les malades du Covid. Deux services ont attiré sur eux l'attention du public, des responsables politiques et des directions hospitalières : d'une part, la réanimation dont les capacités d'accueil sont devenues un enjeu crucial et un indicateur de l'évolution de l'épidémie et, d'autre part, les urgences qui sont passées du statut de guichet contraint par la pénurie de lits à celui de voie d'entrée privilégiée dans un service public vidé de ses usagers et entièrement tourné vers l'accueil des malades du Covid :

« [La crise du Covid], ça nous a donné plus de fluidité, ça glissait, c'est comme si les choses s'imbriquaient de façon naturelle sans qu'on nous dise : "Tu ne peux pas le faire parce que la direction ne veut pas." Là, c'était une espèce d'aisance de mouvement, on était à l'aise dans notre costume... D'un seul coup, c'est comme si les gens avaient découvert l'hôpital et son fonctionnement et tout nous était autorisé. Ça, c'était vraiment un grand changement. »

Stéphanie, infirmière, services des urgences, hôpital B, 8 octobre 2020.

Pour cette infirmière ayant 18 ans d'ancienneté au service des urgences, l'épidémie de Covid a représenté un moment à part, au cours duquel ont été résolus les principaux dysfonctionnements qu'elle perçoit du système hospitalier. Considérant que sa mission principale est de prendre soin de la population, elle supporte de plus en plus mal de voir des patients attendre sur un brancard pendant plusieurs heures. Or, c'est ce type d'anomalie qui a provisoirement disparu. Pendant les quelques semaines de crise épidémique, les urgences ne représentent plus cette part d'impondérable que les autres chefs de service répugnent à prendre en charge en raison d'un nombre insuffisant de lits et d'un fonctionnement à flux tendu difficilement gérable au quotidien :

«Un urgentiste est un médecin qui accueille des patients qui se présentent à la porte. L'activité d'un spécialiste est d'organiser une activité la plus réglée possible pour que ça puisse être fonctionnel avec son bloc ou avec son plateau technique, avec les autres intervenants, etc. Donc plus il programme, mieux ça marche. Et dans sa programmation, moi 
[l'urgentiste] je suis celui qui le fait chier tous les jours, en disant: "Il faut que tu [médecin des services d'hospitalisation spécialisés] sortes des malades plus vite et il faut que tu prennes mes malades." Donc on est des empêcheurs de tourner en rond. Par essence, on les emmerde. »

Thomas, chef de service des urgences, entretien le 26 juin 2020.

La crise sanitaire a ainsi provisoirement enrayé la difficulté des urgences à répartir leurs patients dans les autres services hospitaliers, en raison de l'abondance de lits vidés par la déprogrammation des interventions non urgentes.

\section{Le consentement au surtravail}

En dépit de l'accroissement de la charge de travail, des risques de contamination et des troubles émotionnels liés au grand nombre de décès, les agents se sont investis dans la lutte contre l'épidémie sans compter leur temps ni leur énergie. Le très faible nombre de personnels ayant demandé leur éviction pour raisons de santé en atteste (seulement 42 dans l'hôpital du Grand-Est sur 6500 agents). Loin d'être le fruit d'une pure contrainte de la hiérarchie hospitalière, cet engagement trouve sa source dans un dévouement aux patients très largement partagé, souvent formulé au singulier parmi les motifs de choix des services («le soin au patient», «l'accompagnement de la personne âgée », «le soin au défunt ») et renforcé dans le contexte de crise par la volonté de participer à un morceau d'histoire collective :

« Nous, on arrivait ici à $8 \mathrm{~h}$ du matin et on partait parfois à $21 \mathrm{~h}$, on ne voyait même pas le temps passer. On rentrait, on se douchait et on repartait le lendemain. On faisait comme ça cinq ou six jours d'affilée. Je n'aurais pas pu rester à la maison, il fallait que je vienne, il y avait un truc qui vous appelait à revenir. Et on ne sentait même pas la fatigue. »

Fabienne, brancardière au service mortuaire, hôpital A, 12 novembre 2020.

Le sentiment d'être appelé au travail et d'être pris au jeu s'apparente à une forme d'illusio (Bourdieu, 2003 [1997]) spécifique au champ hospitalier dont le principe structurant est le dévouement aux malades. Sa récurrence dans les paroles d'agents hospitaliers de tous les métiers et de tous grades laisse penser que cet engagement résulte d'un impératif professionnel intériorisé de longue date et largement partagé. Comme dans beaucoup de situations de travail contemporaines, les moyens coercitifs n'ont joué qu'un rôle secondaire par rapport à la valorisation d'un intérêt d'entreprise présenté comme un intérêt universel (Burawoy, 2015, p. 150). Dans un contexte où toute l'attention du débat public était concentrée sur les capacités de résistance des hôpitaux, l'attachement aux collectifs de travail et à la mission de soigner ont beaucoup favorisé le consentement au surtravail, nourri aussi par la dimension collective de la mobilisation.

\section{La mobilisation collective autour d'un objectif commun}

Dans les deux hôpitaux étudiés, la mobilisation autour de l'accueil des malades qui affluaient a été source de cohésion entre les différentes catégories de personnels qui y travaillent :

«Il y avait une espèce de cohésion commune à tous, ça allait de la direction aux femmes de ménage, c'était incroyable. Personne ne se posait la question, tout le monde donnait un coup 
de main à tout le monde, chose [qui] maintenant [...] s'efface parce qu'on revient dans le protocole. »

Mickaël, brancardier, hôpital A, 12 novembre 2020.

Pour ce brancardier qui est passé par l'armée et qui a longtemps été attaché au sentiment de camaraderie qui y régnait, la mobilisation contre l'épidémie a permis de rompre avec un quotidien où chacun est à sa place et s'interdit de s'immiscer dans le travail des autres. S'il est formulé différemment selon la position occupée dans l'organisation hospitalière, ce sentiment d'une mise en suspens des conflits et des concurrences, au profit d'un objectif commun, est largement partagé :

«Il y avait un travail d'équipe sur tout l'hôpital qui était impressionnant, extraordinaire, exceptionnel. En un jour, on a réussi à accorder tous les médecins, tous les services, un truc qui devrait être normal et qui ne l'est pas au quotidien, c'est quelque chose qu'on essaie de faire depuis des années, des années, des années, si ce n'est des décennies. On n'a jamais réussi à le faire et en un jour... Parce qu'on avait tous ce même objectif. »

Hélène, médecin aux urgences, hôpital A, 12 novembre 2020.

Aux deux extrémités de la hiérarchie hospitalière, on retrouve la conviction d'avoir participé à une même œuvre collective, chacun y attribuant des vertus différentes selon sa position dans la division du travail : là où un brancardier en fin de carrière est sensible à l'affaiblissement provisoire de la hiérarchie verticale entre groupes professionnels, une jeune femme médecin généraliste valorise cette expérience comme un moment de mise en suspens des concurrences internes entre chefs de service.

\section{Le renforcement des collectifs de travail}

La crise épidémique a également eu pour effet de renforcer les collectifs de travail selon des modalités différentes en fonction des services et des statuts. Aux urgences et en réanimation, la cohésion des équipes au plus fort de la crise pouvait aller des aides-soignantes aux médecins, avec parfois des tensions entre équipes de jour et équipes de nuit. Dans d'autres services, les collectifs considérés peuvent se limiter parfois aux infirmières ou aux aides-soignantes, par opposition aux cadres et aux médecins. Dans l'hôpital de région parisienne, les médecins du service de réanimation, dirigé par un professeur distant par rapport à ses équipes, n'ont pas le sentiment d'avoir vécu un moment fort de solidarité au travail, tandis qu'aux urgences l'implication de son responsable a beaucoup compté pour préserver une certaine cohésion.

«J'ai l'impression que tout le monde travaillait en même temps et dans le même but. Les médecins des étages ne râlaient pas, les équipes des étages ne râlaient pas non plus, tout était fait de manière fluide, on avait du matériel si on en avait besoin, on avait des cadres présents, on avait la pharmacie qui se déplaçait tous les jours pour gérer nos stocks de sédation pour endormir les gens, nos stocks lambda de médicaments qu'on utilisait tous les jours. On était un peu déchargé pour vraiment se concentrer sur le patient. Pour ça, c'était top. »

Anabelle, infirmière, service des urgences, hôpital A, 15 octobre 2020.

Pour cette infirmière recrutée en 2009 et passée par de nombreux services (pneumologie, psychiatrie) avant d'arriver aux urgences, la crise épidémique a constitué un moment 
d'effervescence où chacun pouvait travailler à sa place en solidarité avec les autres. Dans ce contexte, les cadres de proximité, qui sont d'anciennes infirmières ayant été promues pour coordonner l'activité des infirmières et des aides-soignantes, ont joué un rôle central. Habituellement chargées de gérer les emplois du temps et de veiller à ce qu'il n'y ait pas de retard dans la prise en charge des patients et dans l'acheminement des produits nécessaires, elles ont contribué à organiser les services en filières Covid et non Covid, à rassurer les agents les plus inquiets, à affecter les renforts, à faciliter les échanges avec les autres services et surtout à insuffler des formes d'entraide au sein d'un même collectif de travail. Durant l'épidémie, ce sont elles qui transmettaient aux infirmières et aux aides-soignantes les instructions et les protocoles qui, dans certains cas, changeaient d'un jour sur l'autre. Les variations incessantes de procédures ont accentué la défiance à l'égard des autorités lointaines, telles que le ministère de la Santé et ses antennes régionales (agences régionales de santé [ARS]), mais ont aussi renforcé la solidarité au sein des collectifs de travail de chaque service.

Cette solidarité entre agents s'est également manifestée par des formes d'entraide face au risque de la maladie. Par exemple, au sein du service mortuaire de l'hôpital du Grand-Est, les deux plus jeunes brancardières ont pris en charge les transports les plus exposés aux urgences, pour préserver leurs collègues plus âgées. La cohésion au sein de cet hôpital a aussi été renforcée par la coupure avec le monde extérieur, pouvant même donner le sentiment paradoxal à beaucoup de soignants d'être mieux protégés à l'intérieur de l'hôpital, au chevet des malades et aux côtés de leurs collègues, qu'en ville, au milieu des personnes inconscientes des risques de l'épidémie.

Le sentiment de lutter pour un objectif commun dans le cadre d'équipes solidaires explique en grande partie le consentement au surtravail de la plupart des agents impliqués dans la lutte contre l'épidémie. Cette large adhésion, produit d'une illusio par laquelle chacun est pris au jeu, n'empêche pas la persistance des intérêts divers, voire opposés, des différents joueurs (Bourdieu, 2003 [1997], p. 182). Les hiérarchies entre services et entre catégories de personnels sont loin d'avoir disparues. La crise épidémique s'est encastrée sur des rapports de domination déjà existants, tout en les transformant.

\section{L'encastrement de l'épidémie dans les rapports de domination}

Beaucoup des conflits qui traversent habituellement l'institution hospitalière ont été atténués par la mobilisation générale, mais d'autres ont émergé, notamment autour des risques d'exposition à la maladie. Si la crise épidémique a relégué les logiques bureaucratiques et comptables au second plan, elle n'a pas pour autant mis les agents hospitaliers sur un pied d'égalité. Selon les services et selon les grades, tous les agents n'ont pas bénéficié des mêmes protections et ces différences ont ravivé, voire parfois exacerbé, des rapports de domination qui existaient avant la crise.

\section{Le clivage entre les catégories de personnels en situation de pandémie}

La nécessité de réorganiser les services et les activités pour répondre aux enjeux de la pandémie n'a pas été vécue de la même façon par les médecins, d'une part, et par les infirmières, les aides-soignantes et les secrétaires, d'autre part.

Les médecins dont les services ont été fermés en raison des déprogrammations de consultations ont pu choisir la forme que pouvait prendre leur investissement dans la lutte contre la maladie. En revanche, secrétaires, infirmières et aides-soignantes ont pour beaucoup été assignées à de nouvelles affectations, renforçant leur sentiment d'être des «variables d'ajustement». Décrite par le terme euphémisé de «prêt de personnels entre services », cette pratique existait déjà avant la 
crise épidémique et permettait habituellement aux directions hospitalières de gérer les effectifs à flux tendus. Élaborée sans consultation des équipes concernées, cette contrainte, induisant parfois des risques d'exposition accrus, a été vécue comme un manque de considération et de reconnaissance :

«Les gens en ont marre de comment ils sont traités, ils disent : “On est des pions." Pendant le Covid, c'était un peu ça, on était réquisitionné, on nous mettait là, on nous mettait là. [...] Quand ça manquait, je sais qu'à une période ils prenaient des infirmières de gériatrie pour en mettre en pneumologie... Je peux comprendre que ce ne soit pas facile d'aller dans un service où on n'a jamais travaillé. Et moi je comprends qu'au bout d'un moment les gens en aient marre. On dit que c'est le salaire, c'est vrai qu'on n'est pas forcément bien payé, mais des fois c'est les conditions de travail. On vous appelle n'importe comment : "Il manque quelqu'un, tu viens travailler demain.” »

Annie, infirmière en gériatrie, hôpital A, 24 septembre 2020.

La vision de cette infirmière, qui a été réquisitionnée alors qu'elle avait de l'asthme, de l'hypertension et qu'elle était diabétique, illustre le sentiment d'injustice des soignantes qui se sont trouvées ainsi exposées à la maladie, sans l'avoir vraiment choisi. Craignant pour la santé de ses proches, elle s'est résolue à faire chambre à part avec son mari pendant deux mois, ce qui n'a pas été sans tensions, et elle ne se retrouve d'ailleurs pas dans les récits identifiant la crise à une « parenthèse enchantée ». L'image d'un hôpital uni et rassemblé contre l'épidémie a été davantage relayée par les chefs de service :

«Il y avait une vraie union sacrée dans le but de soulager nos collègues des urgences et de répondre à cette vague, à ce tsunami de pathologies. [...] En fait, c'était une belle expérience humaine, une belle expérience professionnelle dans le sens où on a eu plein de gens qui se sont portés volontaires pour tout de suite donner un coup de main et s'organiser pour la prise en charge. Sur le plan humain et professionnel, c'était très riche. »

Benoit, chef de service d'ophtalmologie, 2 juillet 2020.

Cette représentation irénique de la gestion de l'épidémie reflète une vision largement partagée au niveau des responsables de l'institution. En effet, les réunions d'équipe associant tous les agents ont été vite abandonnées au profit des cellules de crises (Gaudillière et al., 2021, p. 54) rassemblant direction et médecins, ce qui a rapproché temporairement la hiérarchie médicale et l'autorité administrative, mais ce qui a aussi renforcé leur séparation avec les infirmières et les aides-soignantes, soumises à des réorganisations de services et à des réaffectations sur des postes non choisis.

Face à une maladie largement méconnue, contre laquelle il n'existait ni traitement, ni vaccin, beaucoup d'infirmières et d'aides-soignantes ont eu le sentiment d'être directement exposées aux risques de contamination, tandis que médecins, chefs de service et membres de la direction pouvaient davantage garder leurs distances, ce qui n'a pas manqué d'accentuer les divisions internes à l'institution :

«On s'est senti lésé parce qu'on s'est dit : “Ils n'en ont rien à foutre de nous en fait, ils nous donnent des trucs périmés pour travailler." On n'avait pas forcément compris que même périmés, ça fonctionnait. On ne voulait pas l'entendre ça, on voulait des trucs neufs et on nous 
donne des trucs périmés depuis 2001. Pour nous, c'était comme si on nous avait insultés. Après, on ronchonnait dans notre coin, on se disait que la direction, ils étaient en télétravail chez eux... On n'était pas content mais, comme on est des gens intelligents, on s'est dit qu'on n'allait pas faire de grève, parce que la France a besoin de nous. »

Léa, aide-soignante aux urgences, hôpital B, 20 novembre 2020.

Dans l'hôpital de région parisienne, l'absence de visite de la direction dans les services durant la crise épidémique a accentué le clivage entre d'une part les personnels impliqués sur le terrain et exposés à la maladie et d'autre part les agents de l'administration dont le rôle n'était pas toujours immédiatement visible.

$\mathrm{Au}$ sein des équipes, la perception de l'épidémie n'a pas toujours été la même. Pour les médecins, le nouveau virus, encore inconnu quelques mois auparavant, est d'abord apparu sous les traits d'un défi exaltant, avant de susciter une certaine lassitude en raison du caractère stéréotypé et répétitif de sa prise en charge. Pour les infirmières et les aides-soignantes, le coronavirus a davantage été perçu comme une menace, en raison de leurs contacts corporels quotidiens avec les malades. Le sentiment d'abandon a été d'autant plus fort lorsque les directions administrative et médicale semblaient indifférentes à la dureté des conditions de travail. Il n'en est pas de même dans l'hôpital du Grand-Est où les équipes du service mortuaire, durement affectées par les décès, ont ressenti les visites de la directrice du groupe hospitalier comme une forme de reconnaissance d'un travail moins valorisé que les urgences ou la réanimation.

La crise épidémique n'a pas fait disparaître les oppositions entre personnels ; elles ont même été accentuées par la profusion de consignes de prévention qui ont été parfois contradictoires et très inégalement diffusées selon les services.

\section{La perception des consignes de prévention et de leurs variations}

Beaucoup des recommandations diffusées auprès des personnels soignants émanaient des services d'hygiène mais certains chefs de service n'ont pas toujours accepté facilement cette immixtion extérieure. Dans un premier temps, l'illégitimité de ces préconisations liées à l'hygiène a été redoublée par la suspicion que les consignes relatives au port du masque soient en réalité liées à l'insuffisance des stocks. À ce soupçon initial s'est ajoutée une succession de changements de consignes qui a été diversement interprétée par les personnels.

Les médecins et les cadres administratifs ont plutôt perçu ces changements de consignes comme la preuve que l'institution hospitalière était en phase avec les découvertes scientifiques qui surgissaient au fil de la crise épidémique. Les infirmières, les aides-soignantes et les manipulateurs radio étaient en revanche beaucoup plus sensibles à l'insuffisance des stocks de matériels de protection et à la question du risque de transmission. Ainsi, en service de radiologie, les médecins interprétaient les examens radiologiques dans leur bureau sans voir les patients, alors que les manipulateurs radio tentaient d'obtenir des masques FFP2 en raison de leurs contacts rapprochés avec les patients lorsqu'ils les installaient sur la table d'examen radiologique. Au service mortuaire, la consigne a d'abord été de ne pas rouvrir les housses des défunts une fois leur décès constaté, au risque de laisser se propager le virus :

«Au début, on nous disait de mettre le défunt Covid en housse, il ne fallait pas ouvrir. Ensuite, on nous a dit qu'on pouvait ouvrir la housse pour que la famille puisse le voir. Ça, j'ai eu beaucoup de mal. C'était quand même dangereux. Si on nous dit que tout doit être sous housse, on se dit qu'il y a quand même une raison... Je comprenais le côté famille qui 
voulait encore voir leur proche, je comprends tout à fait, mais je voyais aussi qu'on se mettait en danger, nous et notre entourage... On en a beaucoup parlé entre nous, personne n'était vraiment d'accord mais, après, on a suivi les directives parce qu'au final c'est comme ça que ça marche. »

Alexia, brancardière, service mortuaire, hôpital A, 12 novembre 2020.

Dans l'ensemble de ce service, les consignes relatives aux housses ont fait naître des débats autour d'un dilemme moral difficile à trancher: d'un côté, la demande des familles d'ouvrir la housse pour voir une dernière fois les défunts et, de l'autre, la peur des soignantes d'être contaminées par ce biais et de mettre en danger la santé de leurs proches. Cette agente de service de 27 ans, qui a dû attendre six ans avant d'être titularisée sur son poste, s'en est finalement remise à la consigne de la cadre, avec toutefois le sentiment de risquer de mettre en danger sa santé. Les doutes et les critiques quant aux consignes changeantes et contradictoires révèlent la forte sensibilité des agents au risque de contamination, très variable selon les services.

\section{Des niveaux de contamination variables selon les services}

L'exposition à la contamination est très vite devenue un enjeu central, étroitement dépendant de la proximité avec les malades et des protections dont disposaient les soignants.

Au niveau national, parmi les soignants travaillant au sein de l'hôpital public, les taux de contamination selon les catégories de personnels sont relativement proches : en novembre 2020, $14,4 \%$ des aides-soignantes déclarent avoir été testées positivement au coronavirus, alors que ce taux est de $14,5 \%$ pour les infirmières et de $13 \%$ pour les médecins ${ }^{8}$.

Outre les possibles contaminations durant les pauses et les moments de sociabilité, la légère surexposition des infirmières à la maladie peut être imputée à la pratique de gestes à risque de contamination (aspiration bronchique, traitement par aérosols), celle des aides-soignantes à la fréquence et l'intensité des contacts avec les corps (toilette, portage, nettoyage des chambres).

Les différences d'exposition à la contamination apparaissent en revanche beaucoup plus importantes quand on compare les services entre eux. Un premier clivage oppose les services prioritaires en période pandémique, comme les urgences et la réanimation, aux services moins spécialisés, comme la gériatrie. À l'hôpital public, les actions les plus valorisées et les plus exaltantes sont associées à la mission de sauver des vies, tandis que tout ce qui rapproche l'activité hospitalière du travail ouvrier (manutention, manipulation, transport de corps vivants ou morts et de matériel) est habillé de catégories morales telles que le dévouement, le soutien ou l'aide (Peneff, 1992, p. 218). Le second principe de hiérarchisation oppose l'entité principale, concentrant les plateaux techniques et les services de pointe (réanimation, radiologie, chirurgie lourde), aux unités périphériques. En effet, dans chacun des deux sites étudiés, plusieurs structures de soins ont été regroupées ou fusionnées pour devenir les entités périphériques d'un même groupe hospitalier.

Confrontée à d'importantes difficultés d'approvisionnement en masques, la direction de l'hôpital du Grand-Est (A) a très vite été contrainte de rationaliser la distribution de masques :

«On a commencé à avoir des dotations du circuit étatique mais des dotations qui étaient loin de correspondre à notre besoin en volume. [...] C'était compliqué mais à un moment on a

\footnotetext{
${ }^{8}$ Nous remercions Lena Silberzan de nous avoir communiqué ces résultats calculés sur la base des réponses à la vague 2 (novembre 2020) du questionnaire EPICOV, réalisé sur un échantillon national représentatif de 85000 personnes, dont 3096 professionnels de santé.
} 
fait des dotations par service pour essayer de rationaliser la consommation parce qu'on n'avait pas de vision sur le réapprovisionnement. »

Directrice d'un service technique, hôpital A, 9 juillet 2020.

La nécessité de répartir entre services des masques en nombre insuffisant a induit des différences dans les niveaux de protection: les masques FFP2 ont été réservés aux services exposés et les masques chirurgicaux ont été distribués en fonction des préconisations des services d'hygiène, produisant des écarts dans les niveaux d'exposition à la maladie (tableau 1).

\section{Tableau 1}

Taux de contamination selon les services dans l'hôpital A

\begin{tabular}{|c|c|c|c|}
\hline Pôles & Effectifs & $\begin{array}{c}\text { Part de } \\
\text { personnels } \\
\text { testés } \\
(\text { en \%) }\end{array}$ & $\begin{array}{c}\text { Sérologie } \\
\text { positive } \\
\text { (en \%) }\end{array}$ \\
\hline Gériatrie hôpital A2 ${ }^{1}$ & 160 & 93,7 & 44,0 \\
\hline 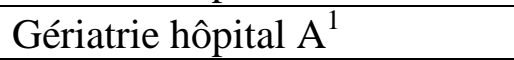 & 307 & 90,6 & 43,2 \\
\hline $\begin{array}{l}\text { Médico-chirurgical } \\
\text { hôpital A1 }\end{array}$ & 123 & 90,2 & 40,5 \\
\hline Gériatrie hôpital A1 ${ }^{1}$ & 144 & 97,2 & 34,3 \\
\hline $\begin{array}{l}\text { Médico-chirurgical } \\
\text { hôpital A3 }\end{array}$ & 41 & 92,7 & 31,6 \\
\hline $\begin{array}{l}\text { Médecine intensive (urgences } \\
\text { réanimation) }\end{array}$ & 465 & 88,6 & 25,2 \\
\hline Gériatrie hôpital A4 ${ }^{1}$ & 181 & 94,5 & 23,4 \\
\hline Maladie rénale et dialyse & 190 & 85,8 & 23,3 \\
\hline$\ldots$ & $\ldots$ & & $\ldots$ \\
\hline Radiologie & 141 & 92,9 & 11,5 \\
\hline $\begin{array}{l}\text { Service des études et de } \\
\text { l'évaluation }\end{array}$ & 51 & 98,0 & 10,0 \\
\hline Pharmacie & 116 & 96,5 & 9,8 \\
\hline $\begin{array}{l}\text { Finances et contrôle de } \\
\text { gestion }\end{array}$ & 111 & 91,9 & 8,8 \\
\hline Anesthésie, bloc opératoire & 326 & 87,4 & 8,4 \\
\hline Périnatalité et gynécologie & 204 & 83,3 & 8,2 \\
\hline Biologie & 151 & 98,0 & 8,1 \\
\hline Ensemble & 5474 & 86,0 & 22,4 \\
\hline
\end{tabular}

1. Les unités A, A1, A2, A3, A4 désignent les différents établissements fusionnés dans un même groupe hospitalier. Pour cet hôpital, nous n'avons pas pu obtenir les taux de contamination par catégorie de personnels.

Source : statistiques réalisées sur la base des résultats des tests sérologiques réalisés auprès de l'ensemble des personnes de l'hôpital A, du 19 au 27 juillet 2020.

La comparaison des taux de positivité selon les services reflète la plus ou moins grande exposition des personnels à la maladie et les différences de dotation en matériels de protection. Dans le Grand-Est (tableau 1), ce sont les services de gériatrie des hôpitaux périphériques (A1, A2 et A3) qui ont été les plus massivement touchés ; les services de médecine intensive (urgences et réanimation) apparaissent également exposés mais de façon moindre ; la forte exposition du service de dialyse (23,3\%) peut être imputable au fait qu'il ait été confronté à des patients qui entrent et qui sortent régulièrement de l'hôpital, en étant parfois transportés à plusieurs dans un même véhicule 
faisant fonction d'ambulance. Enfin, les services administratifs, pharmaceutiques et biologiques sont ceux qui ont été les moins touchés. Cette hiérarchie s'explique en partie par les différences d'exposition aux malades et de dotation en matériels de protection. Dans le cas d'une maladie nouvelle comme le Covid-19, le risque d'exposition peut également provenir de la méconnaissance des formes de contamination, notamment par aérosols. Certains services, comme la réanimation ou les urgences, ont rapidement été approvisionnés en masques FFP2, car l'intubation (par les médecins) et l'aspiration des sécrétions bronchiques (par les infirmières) ont été identifiées très tôt comme des gestes comportant un risque élevé de contamination. En revanche, les infirmières et les aides-soignantes des services où de tels gestes sont rares, voire inexistants, ont mis plus de temps à recevoir le même matériel et à connaître les modes de transmission de la maladie :

«La gériatrie, ça a été un secteur qui a été très impacté parce que c'était un secteur Covid, avec des personnes âgées qui n'avaient pas accès à la réanimation. À l'époque, le personnel n'avait pas de masques FFP2 parce que ce n'était pas recommandé au début et on avait des techniques de traitement de souffrance respiratoire par une ventilation qui était peut-être génératrice de contamination. On s'est rendu compte assez rapidement que la façon de ventiler les personnes qui étaient en difficulté pouvait créer éventuellement des aérosols. [...] Et puis, il y avait énormément de Covid dans les services de gériatrie. Le personnel, tout au début, est tombé malade très vite. »

Médecin du travail, hôpital A, 18 septembre 2020.

Les traitements par aérosols ont été très largement utilisés dans les services qui ont pris en charge les malades ne remplissant pas les critères d'âge et d'autonomie pour être admis en réanimation. Cela explique les taux de contamination très importants dans les services de gériatrie, qui apparaissent alors comme une filière de relégation médicale (Chauvenet, 1978), de surcroît dans les entités périphériques.

Cette différence entre services a été fortement ressentie par les personnels concernés. À la conviction d'appartenir à un service peu reconnu par la direction hospitalière s'est ajouté le sentiment d'injustice d'avoir été exposé à la maladie sans bénéficier des protections suffisantes.

\section{Conclusion}

L'épidémie de Covid a constitué un révélateur des manques et des contradictions d'une institution hospitalière astreinte depuis plusieurs années à des restrictions budgétaires et obnubilée par l'équilibre des comptes. Les routines et les logiques de fonctionnement, découlant des récentes réformes, ont été supplantées par la nécessité d'accueillir et de soigner les malades qui affluaient, sans compter ce qu'il en coûtait à l'hôpital public en termes de ressources humaines et matérielles. L'intérêt d'observer ce moment critique, non pas du point de vue des administrations centrales ou du pouvoir politique, mais depuis l'expérience des personnels hospitaliers, est de mettre en lumière les ressorts d'un service public confronté à l'imprévu et à des besoins qui le dépassent : ce qui a permis à l'hôpital public de maintenir un niveau de fonctionnement minimal face à la crise épidémique, c'est le surtravail des personnels et leur consentement à s'investir dans leur mission bien au-delà de ce que prévoient les règlements. Cet engagement trouve sa source dans l'intériorisation du dévouement aux patients, mais s'explique aussi par la vigueur de collectifs de travail qui ont porté les agents, au point parfois de leur faire renoncer à certains conforts de leur vie personnelle et familiale. 
La comparaison de deux terrains situés dans deux régions différentes montre, d'une part, que les «institutions intermédiaires de l'État » (Durkheim, 2020 [1900]) que sont les hôpitaux publics peuvent jouer un rôle différent selon chaque contexte local et, d'autre part, que la distance différentielle des directions administratives aux services enquêtés détermine fortement l'expérience que les agents ont de l'épidémie, ainsi que leur rapport à l'État et à ses institutions intermédiaires. Dans les services des deux hôpitaux où les personnels de direction ont été peu présents durant toute la crise épidémique, cette distance a renforcé les agents dans leur conviction que cette hiérarchie lointaine était coupée des réalités du terrain au même titre que les autres institutions étatiques (comme Santé publique France ou les Agences Régionales de Santé) pourvoyeuses d'instructions parfois contradictoires. En revanche, dans l'hôpital du Grand-Est, l'énergie déployée par la direction pour trouver des masques et du matériel, à une époque où les administrations centrales se souciaient peu d'une région aux avant-postes de la crise épidémique, a convaincu l'ensemble des personnels que la direction hospitalière était de leur côté. Les critiques à l'égard de représentants de l'État indifférents aux réalités locales ont constitué le ferment d'une solidarité territoriale qui, le temps de la crise épidémique, a permis de dépasser les divisions internes.

L'analyse de ce moment critique est aussi riche d'enseignements si on le replace sur une plus longue période. L'épidémie de Covid et ses «vagues» successives, de plus en plus rapprochées, exigent du personnel hospitalier un surcroît de travail durable et récurrent, sans que la reconnaissance des pouvoirs publics soit à la hauteur des efforts consentis. Les hausses de salaire décidées par le gouvernement en juillet 2020 restent bien en deçà des attentes des agents, qui espéraient des réformes structurelles améliorant leurs conditions de travail et plaçant leur mission de soin au cœur de l'institution hospitalière. En plus de s'être mobilisés, sans compter leurs forces et leur énergie, durant toute la crise épidémique, les agents ont eu, une fois la crise passée la conviction d'avoir travaillé sans compter aux yeux de l'administration hospitalière et étatique, c'està-dire sans que leurs efforts soient reconnus. Dès lors, ce qui était supportable avant la crise épidémique ne l'est plus, les départs d'infirmières vers le secteur libéral et de médecins vers les cliniques privées se multiplient, de façon plus marquée dans le Grand-Est, laissant les directions hospitalières coincées entre la nécessité d'accueillir les malades et l'impossibilité de pourvoir les postes. Le moment critique qu'a été la crise épidémique pourrait alors devenir un tournant après lequel la croyance dans le service public ne suffit plus à faire fonctionner une institution exsangue.

\section{Références bibliographiques}

Belorgey N. (2010), L'hôpital sous pression: enquête sur le "nouveau management public », Paris, La Découverte.

Bourdieu P. (1984), Homo Academicus, Paris, Minuit.

Bourdieu P. (2003 [1997]), Méditations pascaliennes, Paris, Seuil.

Burawoy M. (2015), Produire le consentement, Paris, La ville brûle.

Chauvenet A. (1978), Médecines au choix, médecine de classes, Paris, Presses universitaires de France.

Collectif Inter-Urgences (2021), «L'urgence de se faire entendre », Actes de la recherche en sciences sociales, $\mathrm{n}^{\circ} 236-237$, p. 92-101.

Durkheim É. (2020), Sociologie politique : une anthologie, Paris, Presses universitaires de France.

Gaudillière J.-P., Izambert C., Juven P.-A. (2021), Pandémopolitique. Réinventer la santé en commun, Paris, La Découverte. 
Juven P.-A., Lemoine B. (2018), «Politiques de la faillite», Actes de la recherche en sciences sociales, $\mathrm{n}^{\circ} 1$, p. 4-19: https://www.cairn.info/revue-actes-de-la-recherche-en-sciences-sociales2018-1-page-4.htm

Juven P.-A., Pierru F., Vincent F. (2019), La casse du siècle. À propos des réformes de l'hôpital public, Paris, Raisons d'agir.

Morel S. (2019), « Inequality and discrimination in access to urgent care in France. Ethnographies of three healthcare structures and their audiences », Social Science \& Medicine, ${ }^{\circ} 232$, p. 25-32.

Peneff J. (1992), L'hôpital en urgence : étude par observation participante, Paris, Métailié.

Pierru F. (2020), « Introduction. L'administration hospitalière, entre pandémie virale et épidémie de réformes », Revue française d'administration publique, vol. 2, n 174, p. 301-315.

Vincent F. (2017), «Penser sa santé en travaillant en 12 heures. Les soignants de 1'hôpital public entre acceptation et refus », Perspectives interdisciplinaires sur le travail et la santé, 19-1: https://journals.openedition.org/pistes/4945 